\title{
Study of a noncontact type micro-CMM with arch-bridge and nanopositioning stages
}

\author{
Kuang-Chao Fan ${ }^{\mathrm{a}, \mathrm{b}, *}$, Yetai Fei ${ }^{\mathrm{a}}$, Xiaofen $\mathrm{Yu}^{\mathrm{a}}$, Weili Wang ${ }^{\mathrm{a}}$, Yejin Chen ${ }^{\mathrm{a}}$ \\ ${ }^{\mathrm{a}}$ School of Instrument, Hefei University of Technology, Anhui, China \\ ${ }^{\mathrm{b}}$ Department of Mechanical Engineering, National Taiwan University, Taipei, Taiwan
}

\begin{abstract}
A high precision micro-CMM (coordinate measuring machine) is under development. The expected measuring range is $25 \times 25 \times 10 \mathrm{~mm}$ and the resolution is about $1 \mathrm{~nm}$. For the enhancement of machine accuracy, some new design concepts are introduced. The arch-bridge is proven to have higher stiffness than the conventional rectangular bridge. The proposed co-planar stage can reduce the Abbé error in the vertical direction. The long travel of each axis is activated by the piezo-ceramic ultrasonic motor with its AC drive mode. The fine positioning is achieved by the same motor with its DC drive mode, which can perform nanometer steps proportional to the input voltages. A linear diffraction grating interferometer (LDGI) is developed as the position feedback sensor with the resolution to $1 \mathrm{~nm}$ after the waveform interpolation. The autofocusing probe is adopted from DVD pickup head and modified to achieve long-range and short-range detections. Using its focus error signals (FES), the probe can reach to $30 \mathrm{~nm}$ accuracy in the short range. In this paper, the design principles of the developed micro-CMM are described.
\end{abstract}

(C) 2006 Elsevier Ltd. All rights reserved.

Keywords: Micro-CMM; Co-planar stage; Arch-bridge; Finite element analysis

\section{Introduction}

The technology of ultra-precision 3-D profile measurement has received much attention during the past decade [1]. Various types of nanometer probes were developed and successfully commercialized, such as STM, AFM, confocal probe, autofocusing probe, etc. [2-5]. It was noted by Takamasu [6] that most researches in the past dedicated interests in the 1-D probe system only. An overall consideration in the 3-D system and its integration should not be overlooked. Small-sized CMMs have become a new topic of research, such as the nano-CMM by Takamatsu [6], small-CMM by NPL [7], miniature probe system by Eindhoven University [8] and PTB [9], the nanopositioning and nanomeasuring machine by Jäger [10], the sub-atomic measuring machine by UNCC/MIT [11], and the microCMM by Fan [12], etc. A small CMM requires higher

\footnotetext{
${ }^{*}$ Corresponding author. Department of Mechanical Engineering, National Taiwan University, Taipei, Taiwan. Tel.: + 886223620032 ; fax: +886223641186 .

E-mail address: fan@ntu.edu.tw (K.-C. Fan).
}

accuracy and resolution. Scaling down the conventional CMM design principle is not a feasible way. Some new design concepts have to be introduced. This paper presents an innovative CMM design, including the arch-bridge, the co-planar precision XY-stage, the spindle, the motion actuator and feedback system, and the autofocusing probe. This micro-CMM is designed for the measurement of mesoscale parts to the accuracy of nanometer range. It is aimed at achieving $1 \mathrm{~nm}$ resolution and $30 \mathrm{~nm}$ accuracy within a measuring range of $25 \times 25 \times 10 \mathrm{~mm}$.

\section{System configuration of the micro-CMM structure}

\subsection{Concept of the arch-bridge structure [13]}

The rectangular type of the bridge is always employed in the precision CMM structure for mounting the $Z$-axis and the probe, as shown in Fig. 1. The deformation in the center of the bridge is very critical because of the concentrated load from the spindle. The maximum deflection at the center of the rectangular-bridge can be 


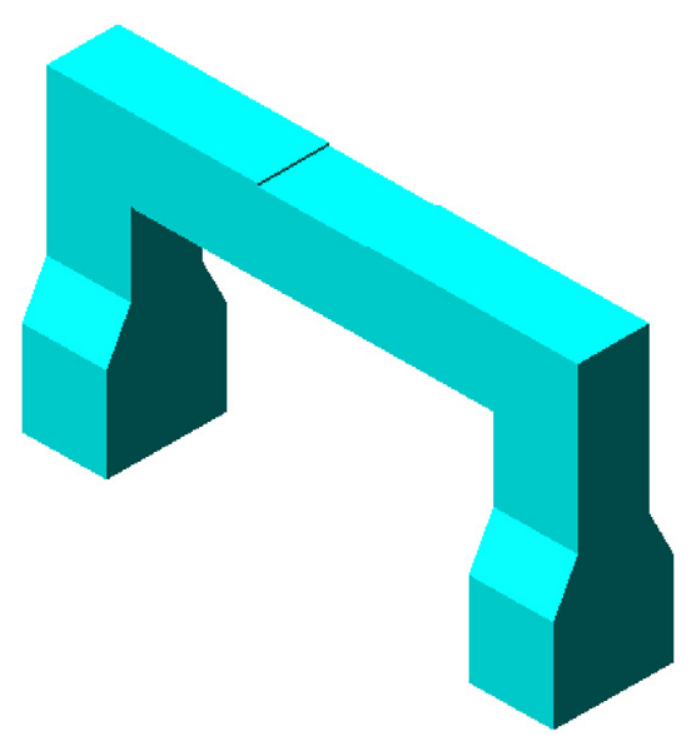

Fig. 1. The conventional rectangular bridge of the CMM.

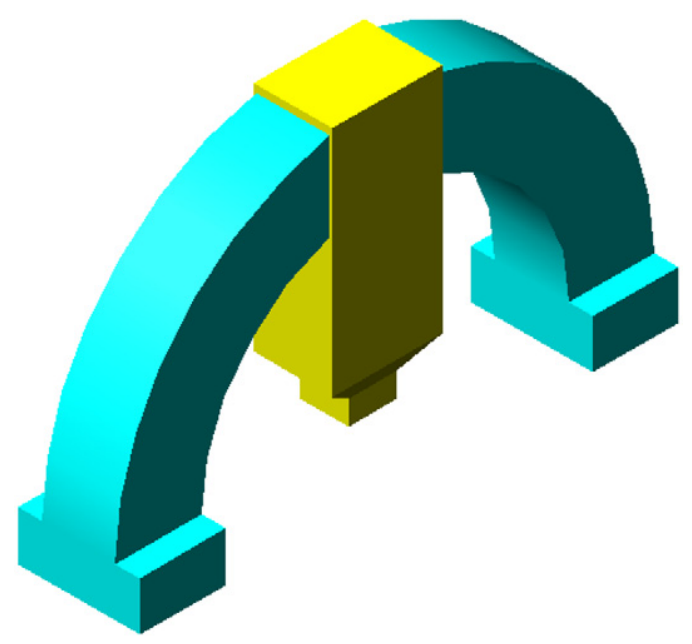

Fig. 2. The proposed arch-bridge with spindle.

described as ( $P$ is the spindle load and $R$ is the half span of the bridge):

$\delta_{y \max }=0.55 \frac{P R^{3}}{E I}$.

The research proposes a fixed arch-bridge structure, as shown in Fig. 2. Under the same dimension and the same spindle load, the maximum deflection at the center of the arch-bridge can be reduced to ( $R$ is the radius of the semicircle bridge):

$\delta_{y \max }=0.24 \frac{P R^{3}}{E I}$.

It can be seen that the arch-bridge performs better static stiffness than the rectangular bridge.

The physical dimensions of the developed micro-CMM bridge are: the outer radius is $220 \mathrm{~mm}$ and the inner is $150 \mathrm{~mm}$; the width is $60 \mathrm{~mm}$ and the dimension of the
Table 1

Comparison of deformation between rectangular-bridge and arch-bridge

\begin{tabular}{llll}
\hline Type of bridge & $\begin{array}{l}\text { Analytical } \\
\text { solution with } \\
\text { spindle load } \\
(\mu \mathrm{m})\end{array}$ & FEA & \\
\cline { 3 - 4 } & & $\begin{array}{l}\text { With spindle } \\
\text { load }(\mu \mathrm{m})\end{array}$ & $\begin{array}{l}\text { Self-weight only } \\
(\mu \mathrm{m})\end{array}$ \\
\hline Rectangular & 0.398 & 0.362 & 0.156 \\
Arch & 0.197 & 0.174 & 0.102 \\
\hline
\end{tabular}

supporting pad is $70 \mathrm{~mm} \times 100 \mathrm{~mm} \times 40 \mathrm{~mm}$. It is made of granite material. The total weight is about $40 \mathrm{~kg}$. The spindle adds additional weight of about $3 \mathrm{~kg}$. Table 1 lists the comparison between the analytical and FEM methods. It can be seen that the arch-bridge performs better static stiffness than the rectangular bridge. Any CMM normally operates in touch-triggered or scanning mode. At the time of taking measuring data the spindle may carry the probe to move in the $Z$-direction. Any driving force exerted by the actuator may also generate reactive force to the bridge structure, which will be dynamically deformed in nanometer range. The FEM analysis shows that for a $0.5 \mathrm{~N}$ driving force in the $Z$-direction, the corresponding additional deformation will be incurred on the rectangular bridge for about 18 and $10 \mathrm{~nm}$ on the arch-bridge. The stiffness of the bridge is, therefore, very essential to the measuring accuracy.

\subsection{Concept of the co-planar $X Y$ stage}

Conventional $X Y$ stage is stacked up by two linear stages composing of many components, such as ball screw, bearing, linear slide, etc., as shown in Fig. 3. The Abbé error of the lower stage is high and the components are made in micrometer accuracy ranges. More considerations should be taken into account when the $X Y$ stage is used for the micro/nanomotion accuracy. An innovative co-planar stage is thus proposed in this study, shown in Fig. 4. The table is moved along the precision ground rod of the frame in the $X$-direction, and the frame is moved in the $Y$-direction along the precision ground rod of the base. Four gliding rods are located in the same plane, as shown in Fig. 5. The $X$-table is embodied in the $Y$-frame and the $Y$-frame is embodied in the base. Such a design can compress the whole stage into a single layer from the side view. It can significantly reduce the Abbé error in the vertical direction. In addition, there are no more transmission components and the geometry is symmetrical, which ensures better static deformation under the same working conditions. Each axis motion is actuated by a motor from one side and its motion is detected by a holographic grating scale from the other side. The moving table is sliding along the guiding rod with a Teflon pad to reduce the friction. The whole stage is made of Invar steel so that the thermal deformation due to the driving heat can be significantly 


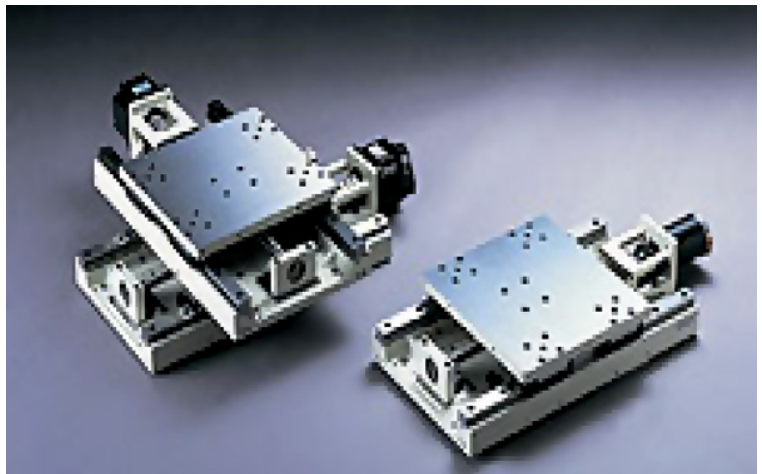

Fig. 3. Conventional $X Y$-stage which is stacked up by two linear stages.

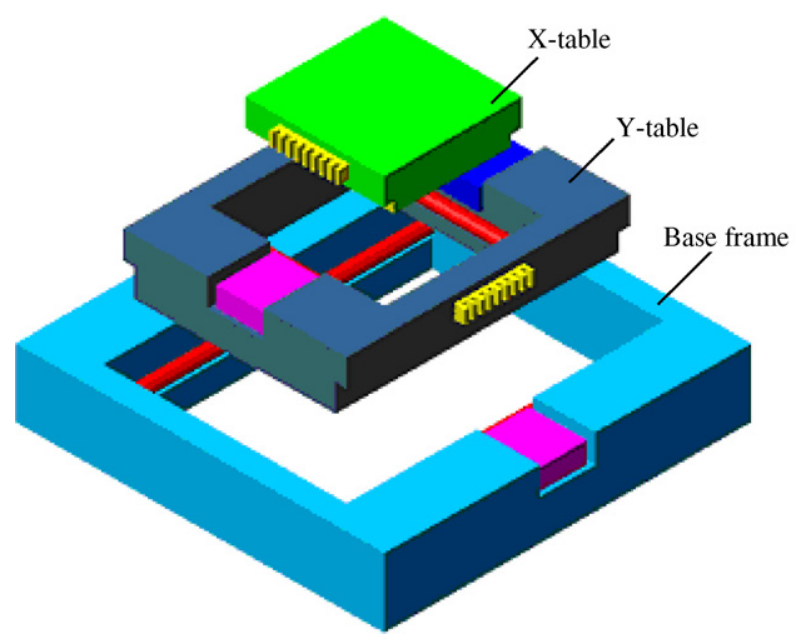

Fig. 4. Proposed co-planar $X Y$-stage in exploded view.

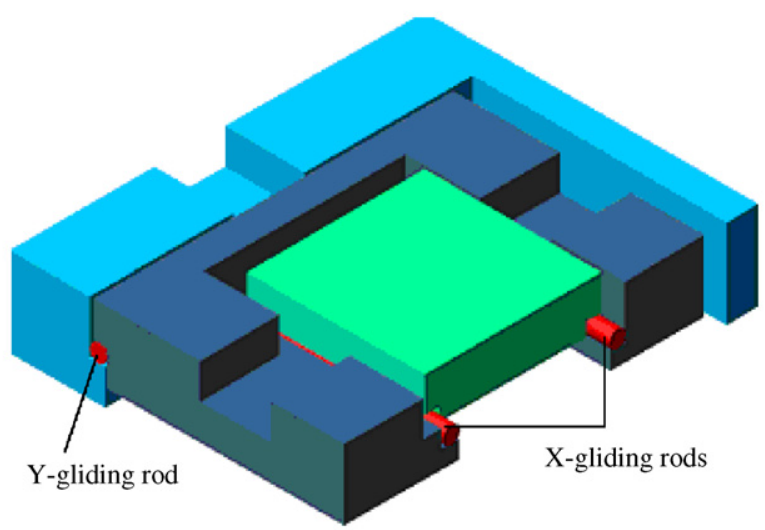

Fig. 5. Sectioning view showing the gliding rods and the single layer from side view.

reduced. In order to minimize the moving weight, a modified structure is designed in the form of Fig. 6. From the finite element analysis with ANSYS software it shows that at the table center the static deformation is about $0.13 \mu \mathrm{m}$, and the thermal distortion is only $3.2 \mathrm{~nm}$ assuming there is a $5{ }^{\circ} \mathrm{C}$ temperature increase at the driver [13].

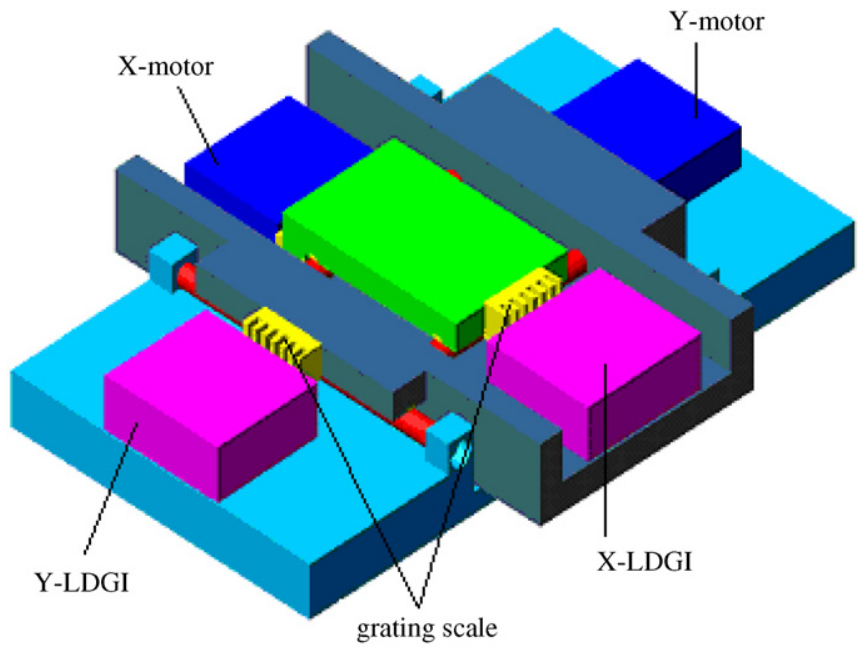

Fig. 6. Final stage design showing all components after weight reduction.

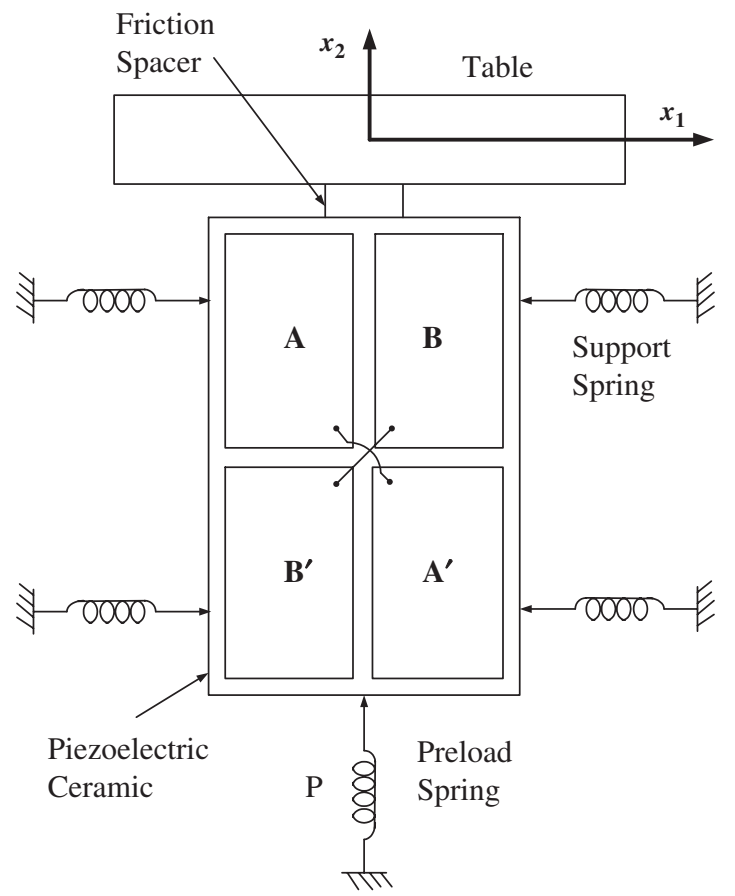

Fig. 7. Structural diagram of the ultrasonic motor composing four PZT pieces.

\subsection{The drive system}

\subsubsection{The long stroke motion}

In order to remain at high motion accuracy, the coplanar $X-Y$ stage and the $Z$-stage are all driven by the attached ultrasonic motor, model HR-4, made by Nanomotion Co. of Israel $[14,15]$. Each HR-4 motor is composed of four piezoelectric ceramic plates (as shown in Fig. 7) and can perform the motion in two modes: $\mathrm{AC}$ and $\mathrm{DC}$ of the friction spacer. The $\mathrm{AC}$ mode is a resonant mode which can drive the stage quickly and realize large displacement in a short time. Four electrodes are adhered to the piezoelectric ceramic structure. Each electrode occupies approximately one quarter of the total surface 
area. The undersurface of the piezoelectric ceramic is covered with a single electrode. Each pair of diagonal electrodes, i.e. $\mathrm{A}, \mathrm{A}^{\prime}$ and $\mathrm{B}, \mathrm{B}^{\prime}$, is connected via an electrical lead, and the electrode of the undersurface is grounded. Giving in-phase or out-of-phase AC signal to each pair of the two components of piezoelectric effect will be generated. This effect converts electrical field to a mechanical motion in both longitudinal and bending vibration modes of the motor, as shown in Fig. 8. The system is designed such that the two vibration modes have a common natural frequency. The resulting composite vibration mode causes the tip of the friction spacer to execute elliptic motions. The friction spacer is appropriately preloaded and is located in contact with a ceramic plate mounted on the moving table. This elliptical motion then drives the stage by friction force to create linear motion, as shown in Fig. 9. Theoretically this AC mode generates $5 \mathrm{~nm}$ displacement of the stage at each cycle. Continuous cycles thus generate continuous long stroke motion of the stage.

\subsubsection{The short stroke motion}

The DC mode of the ultrasonic motor is excited by giving a DC voltage so that the spacer tip can generate a small motion proportional to the input voltage. The range of voltage is from -10 to $10 \mathrm{~V}$, corresponding to the output

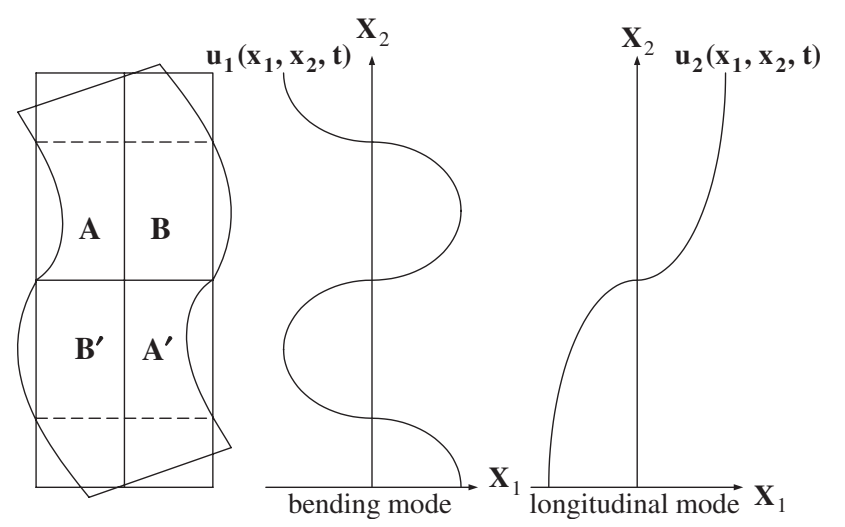

Fig. 8. Longitudinal and bending vibration modes of piezoelectric motor.

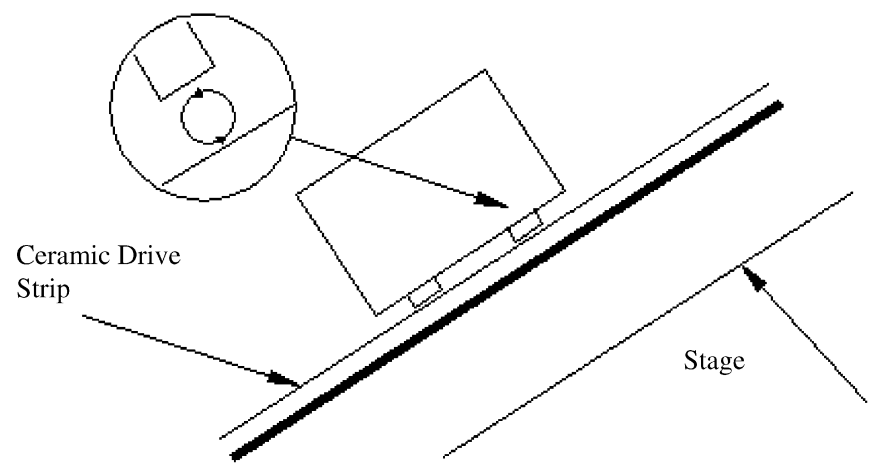

Fig. 9. Motion principle of ultrasonic motor showing the tip in elliptical motion. friction motion of the stage from -400 to $400 \mathrm{~nm}$. With a proper combination of the $\mathrm{AC}$ and $\mathrm{DC}$ modes the stage can perform long travel and fine motion to nanometer resolution. This motion can be controlled with the integration of a position feedback sensor and a PID motion controller.

\subsection{The feedback sensor}

The position feedback of linear motion in each axis is detected by the principle of diffraction interferometry [16]. A linear diffraction grating interferometer (LDGI) is developed with a $1 \mathrm{~nm}$ resolution, as shown in Fig. 10 . The laser diode (1) emits a laser beam with $635 \mathrm{~nm}$ wavelength. In order to reduce the physical dimension of the LDGI system, the laser beam is bent by two mirrors (3) before entering into the gratings (2). The gratings, fabricated by holographic method with $1200 \mathrm{line} / \mathrm{mm}$, will reflect with \pm 1 diffraction beams to two reflecting mirrors (3), respectively. Combining these two beams with a beam splitter (4) and then expanding its diverging angle through a condenser lens (5), the interferogram will fall into the array photodiode (6). Frequency shift of the \pm 1 st order diffraction beams due to the Doppler shift will occur as soon as the grating moves. The actual frequencies of the two diffraction beams can be derived as

$$
\left\{\begin{array}{l}
f_{+1}=f_{0}+\frac{v}{d_{\mathrm{g}}}, \\
f_{-1}=f_{0}-\frac{v}{d_{\mathrm{g}}},
\end{array}\right.
$$

where $f_{0}$ is the frequency of incident light; $f_{+1}$ is the frequency of the +1 st order diffraction beam; $f_{-1}$ is the frequency of the -1 st order diffraction beam; $v$ is the inplane velocity of the grating; $d_{\mathrm{g}}$ is the grating constant.

Thus, the interference fringes created in the photo detector array move as well because of the beating

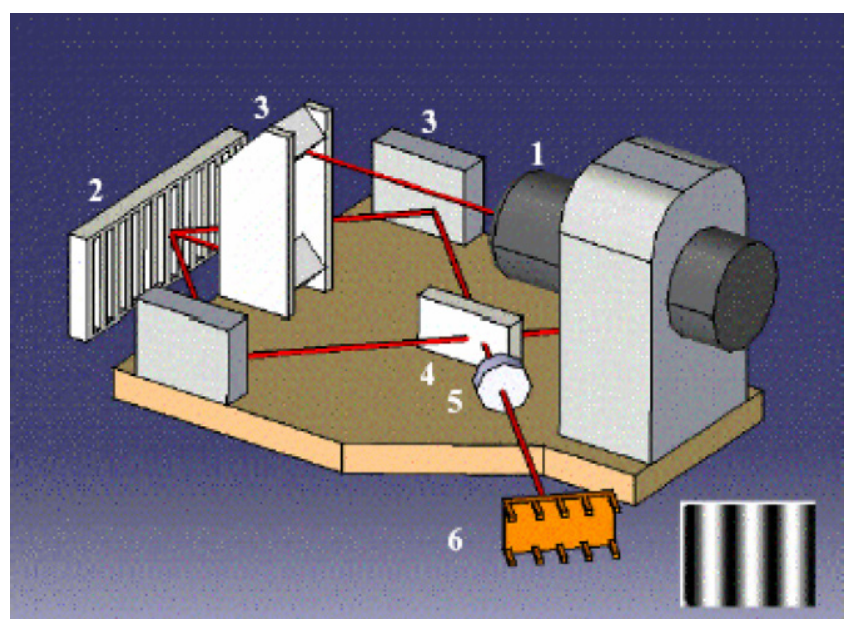

Fig. 10. The principle of LDGI (1: laser diode, 2: gratings, 3: mirror, 4: beam splitter, 5: condenser lens, 6: array photodiode). 
frequency shown as follows:

$\Delta f=f_{+1}-f_{-1}=2 \frac{v}{d_{\mathrm{g}}}$.

The magnitude of the beating signal received by each element of photo detector array can be obtained as

$I(t)=I_{1}+I_{2} \cos \left(2 \pi \int_{0}^{t} \Delta f \mathrm{~d} t\right)=I_{1}+I_{2} \cos \left(4 \pi \frac{\Delta z}{d_{\mathrm{g}}}\right)$,

where $t$ is time and the upper limit of integration is beating signal period $T ; 4 \pi\left(\Delta z / d_{\mathrm{g}}\right)$ is the phase shift due to beating signal when Doppler effect occurs; $I_{1}$ and $I_{2}$ are the magnitudes of DC and AC term of the received signal, respectively.

The drift velocity of the fringe is proportional to the moving velocity of the grating. A period alteration of the fringe alters the beating signal for a cycle. The phase shift for a cycle is equal to $2 \pi$ and the relationship of signal and geometry variation can be expressed as

$2 \pi=4 \pi \frac{\Delta z}{d_{\mathrm{g}}}$.

Thus, the displacement of the grating due to the stage movement and the quantities of pulse due to phase shift from beating signal are correlated as $\Delta z=d_{\mathrm{g}} / 2$. As a result, a signal with two sine-wave periods per grating pitch is obtained from each element of the photo detector array. The photo detector array then converts the interference fringe pattern into a set of four sinusoidal signals that are differing in phase by $\pi / 2$ with each other along the detected moving direction, as shown in Fig. 11. In this study, the photodiode array is used to detect the interference fringe pattern. The amplitude of illumination distribution of interference pattern, along the direction perpendicular to the fringe, is sinusoidal and should be tuned to fit to the size of photodiode element. To get output signals in orthogonal sinusoidal waves, the geometry of the photodiode array elements are placed relative to the interference

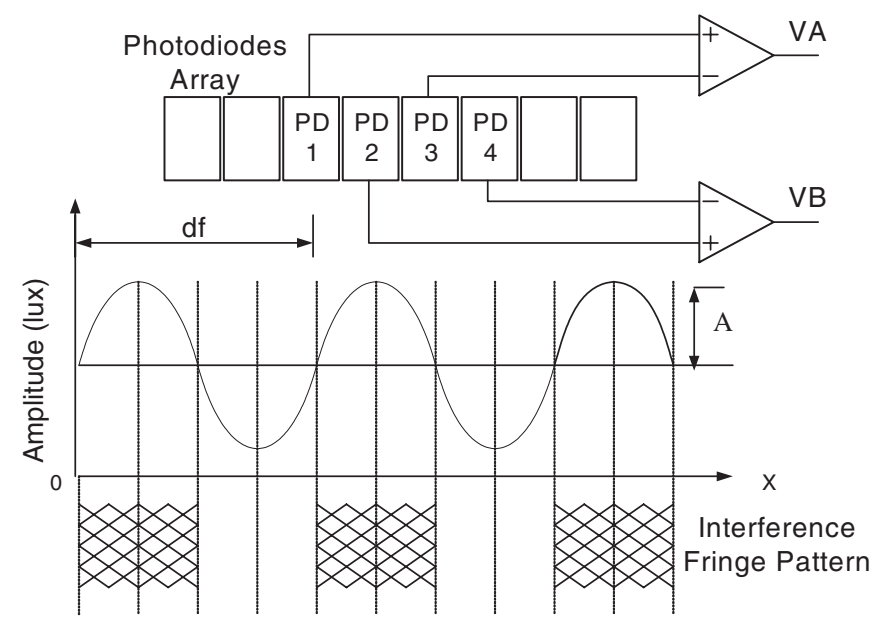

Fig. 11. Schematic of photo detector system showing the relationship between the sinusoidal signal and the four photodiodes. patterns in such way that photo detector element $\mathrm{PD}_{1}$ detects fringe of first interference order. With the operation of $\left(\mathrm{PD}_{1}-\mathrm{PD}_{3}\right)$ and $\left(\mathrm{PD}_{2}-\mathrm{PD}_{4}\right)$ two orthogonal sinusoidal signals with $\pi / 2$ phase-shift can be obtained. Passing through a Schmidt trigger IC these two signals can transform into two square waves.

These two signals with the phase difference of $90^{\circ}$ can be transformed into two phase-shifted square waves. And through a multiplication operation these two square waves can be transformed into another square waves with a doubled frequency. With regard to the fringe subdivision technique, although there have been many methods proposed by various researches, such as the curve fitting [17], signal modulation [18] and with CCD [19], this research developed a simple and quick method of triangulation form approximation by digital computing $|\sin \theta|-|\cos \theta|$ from the output signals, as shown in the bottom of Fig. 12. The complete cycle counting and directional sensing can be done by conventional up/down counter. Only the incomplete cycles at the beginning and the end are to be interpolated. The calculation of $X_{0}$ and $X_{1}$ is very simple. The approximated linearity is about $4 \%$. Each linear range of the triangular form represents one quarter of the sinusoidal wavelength and one-eighth of the grating pitch. The grating pitch is about $833 \mathrm{~nm}$. With a simple $\times 100$ linear interpolation calculation it is easy to reach $1 \mathrm{~nm}$ resolution.

The position feedback sensor equipped in ordinary precision stages is the linear scale, which normally provides the resolution to 1 or $0.1 \mu \mathrm{m}$ in some particular applications, and the accuracy in several microns. This is not adequate enough for nanopositioners. Laser interferometer is the current solution for most ultra precision stages, which require the resolution to $1 \mathrm{~nm}$ and the accuracy in $10 \mathrm{~nm}$ in the extreme temperature-controlled environment. Laser interferometer is sensitive to the variation of ambient

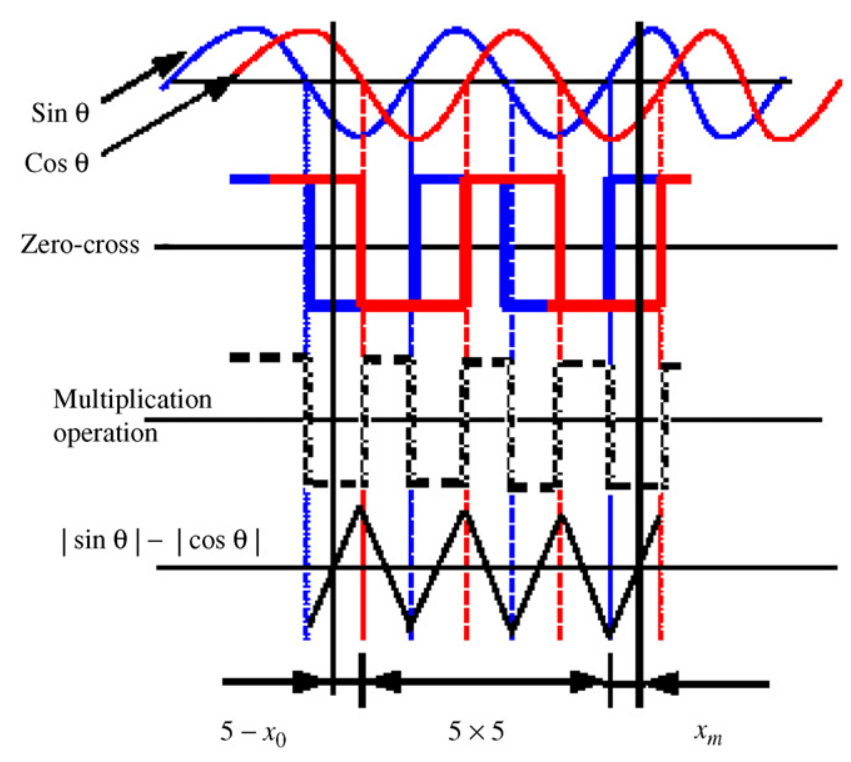

Fig. 12. The fringe counting and subdivision signals. 
conditions as its basic scale, the wavelength, is temperature-dependent. In addition, the laser interferometer is expensive and its size is large in comparison with the size of all fine motion stages. Only the single frequency laser interferometer can be pigtailed by a fiber to reduce its emitting head [20]. The hologram grating scale measures the displacement of the stage based on its scale pitch, which is immune to atmosphere variations. As a result, the grid accuracy can be even better than that of a laser interferometer. The grid has manufacturing imperfections, but they are generally predictable and can be software compensated. Moreover, the grating scale system is very low cost. This is why this system is adopted in this study.

\subsection{Spindle head design}

The spindle that carries the probe is moved along a short linear stage, which is driven by the same ultrasonic motor as used in the co-planar stage, and its motion is detected by a LDGI, as shown in Fig. 13. The grating scale is in line with the spindle and the probe so that there is no Abbe error. A counterweight is applied to balance the total mass center during the spindle motion. The whole structure of the developed micro-CMM is shown in Fig. 14, which contains the granite base, the granite arch-bridge, the Invar co-planar stage, and the $Z$-spindle.

\section{Development of an autofocusing probe}

This research aims at development of a low-cost optical probe with the measurement capability in the sub-micron range. The pickup head of a commercial DVD player was adopted based on its principle of focus error. With the proper modification on its signal processing unit, this head can be converted to a profile probe with sub-micron accuracy.

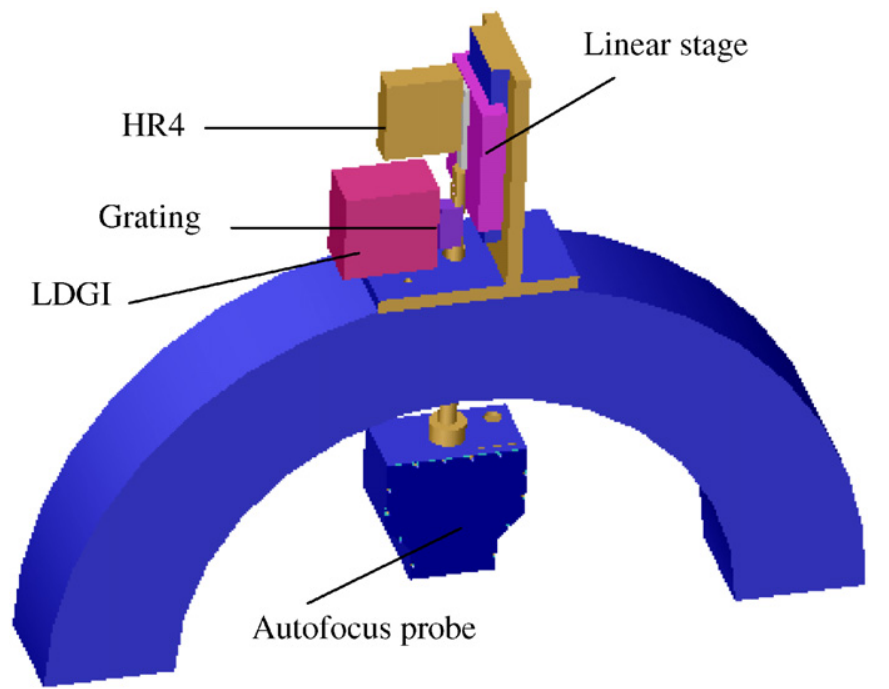

Fig. 13. The composition of spindle head design on the arch bridge.

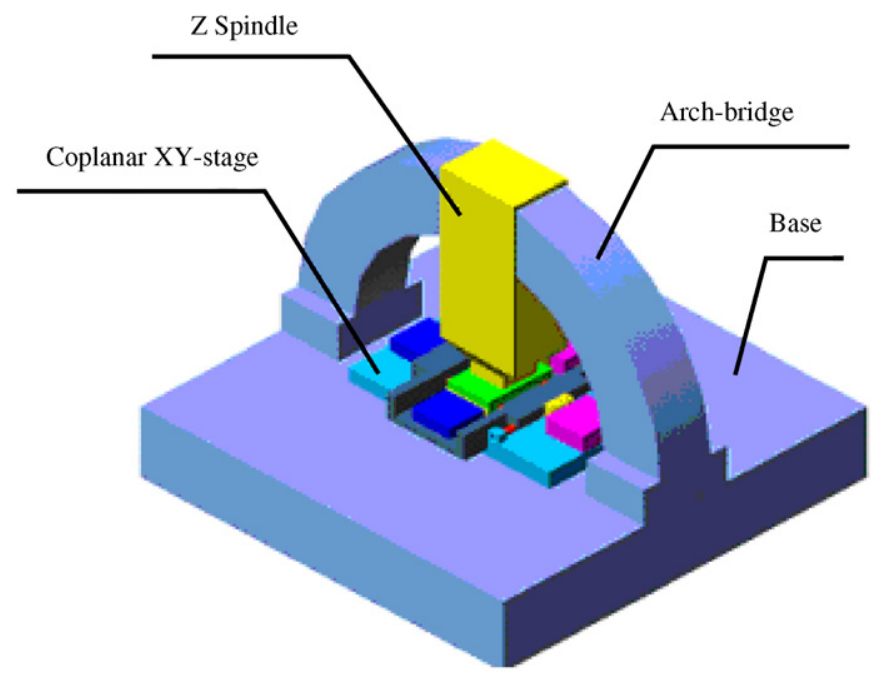

Fig. 14. The configuration of the developed micro-CMM.

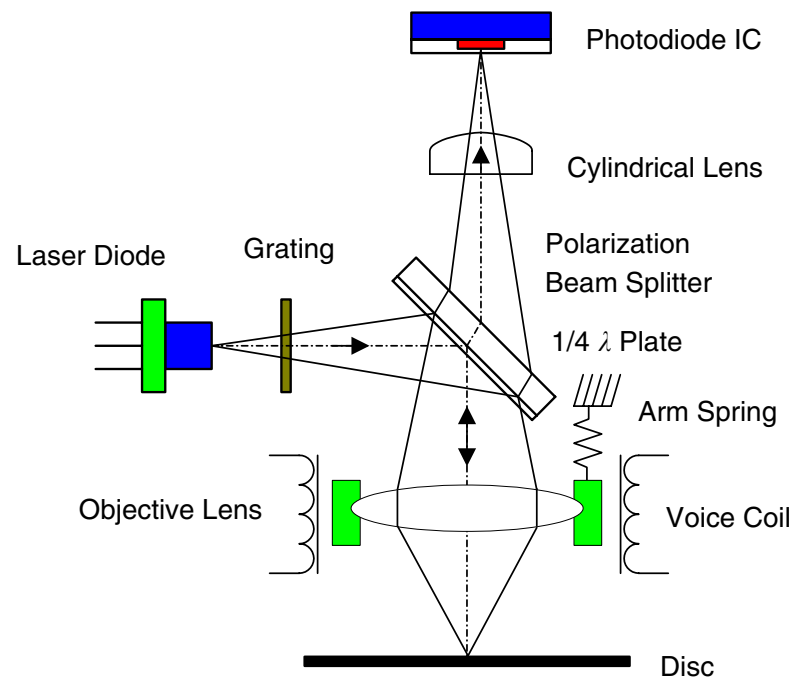

Fig. 15. Principle of autofocusing probe.

The commercial DVD player pickup head was used for this experiment. In principle, the pickup head essentially is an autofocusing laser probe, as shown in Fig. 15. A $650 \mathrm{~nm}$ wavelength light source generated from a laser diode is primarily polarized by a grating plate. By passing through a beam splitter and a quarter waveplate the light beam is focused by an objective lens onto a $2 \mathrm{~mm}$ away object surface with a spot size of approximately $1 \mu \mathrm{m}$ in diameter. The reflected beam signal is imaged onto a four-quadrant photo detector by means of the quarter wave plate. The photodiode outputs are combined to give a focus error signal (FES) which is used for feedback control of the position of the movable lens suspended by the voice coil motor within the sensor such that the focal spot of the beam remains coincident with the object surface. In this system, the focusing signal is detected by the Astigmatic method. At the focal plane, the spot is a pure circle. While away from the focal plane, the spot appears as an elliptical shape in different orientations with inside or outside of the 
focal planes. The corresponding FES is detected by a photodiode, which provides an $S$-curve signal proportional to the distance, as shown in Fig. 16. This motion control strategy is implemented by a DSP (TI Co, 32-bit). The linear range can be extended to over $1 \mathrm{~mm}$, as shown in Fig. 17.

\section{Experimental tests}

The developed micro-CMM has been fabricated in modules and integrated into a prototype machine. Fig. 18 shows the photo of this prototype. The physical dimensions are about: $X-450 \mathrm{~mm}, Y-450 \mathrm{~mm}$, and $Z-320 \mathrm{~mm}$. The $X$ table of the co-planar stage is embodied in the $Y$-stage, and the $Y$-stage is embodied in the base frame. It makes the thickness of the co-planar stage to as thin as about $30 \mathrm{~mm}$. Some performance tests have been carried out.

\subsection{Positioning accuracy tests}

The motion of each axis can be calibrated using a laser interferometer in a temperature- and humidity-controlled room. For the long stroke test with AC mode, the original

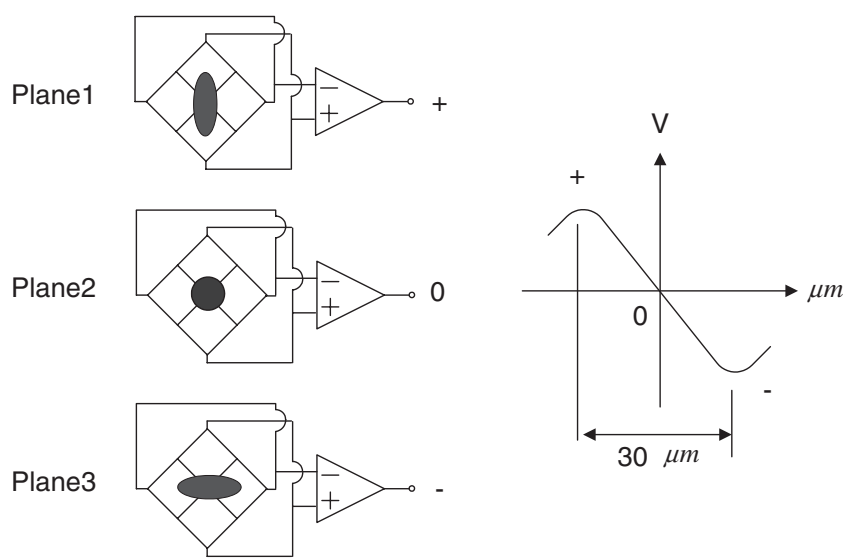

Fig. 16. The spot shape vs. the $S$-curve (Plane 1: surface near to the focus, Plane 2: on focus, Plane 3: away from focus).

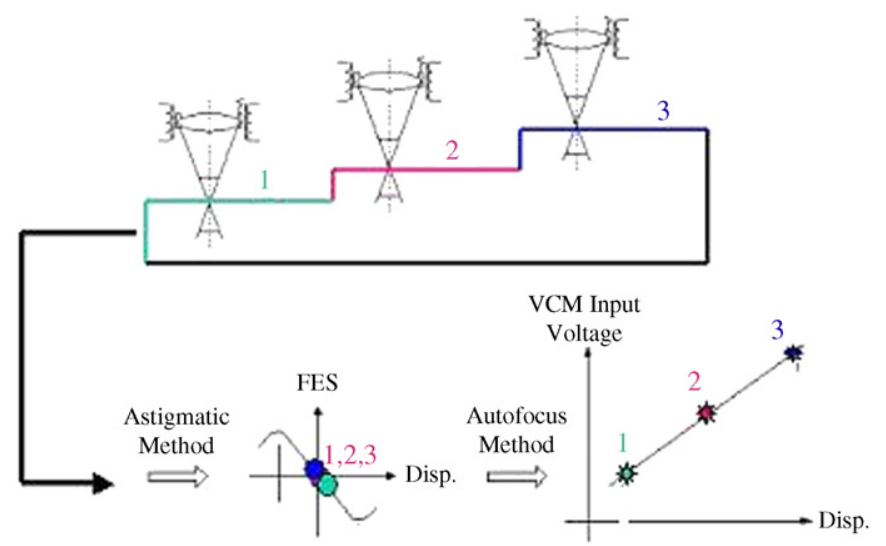

Fig. 17. Autofocusing activation at three different heights. positioning accuracy exhibits inherent systematic errors. Using the error compensation scheme the error map can be reduced to about $0.9 \mu \mathrm{m}( \pm 3 \sigma)$ over $25 \mathrm{~mm}$, as shown in Fig. 19. For the short-range test with only $0.3 \mu \mathrm{m}$ travel (fine motion with DC mode), the hysteresis errors appear in the forward and backward motions. Since the repeatability remains good, separate curve fitting analysis was applied to individual motions. The averaged maximum positioning error is only $20 \mathrm{~nm}$ after error compensation, as shown in Fig. 20. This performance can meet our original requirement of $30 \mathrm{~nm}$.

\subsection{Autofocusing probe tests}

The FES test was carried out by mounting the probe on a linear stage. The stage motion was measured by a laser interferometer. Fig. 21 shows the tested results with respect to different materials. Good $S$-curve means the material has good reflection surface [21]. It can be seen that the CD plate surface performs the best characteristic curve because the probe is designed for this material. The silicon-based material will absorb the light near red to infra red

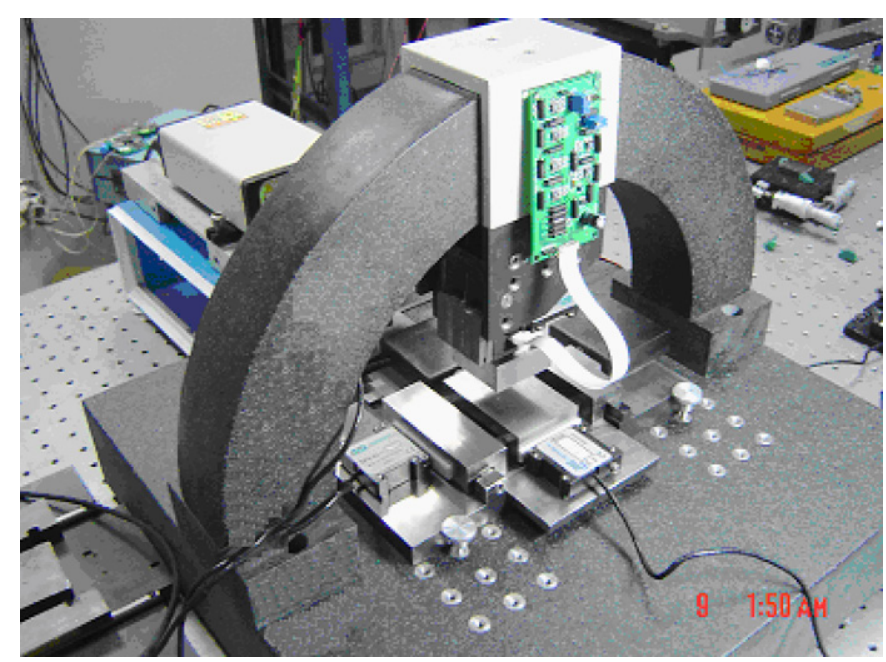

Fig. 18. Photo of the prototype micro-CMM.

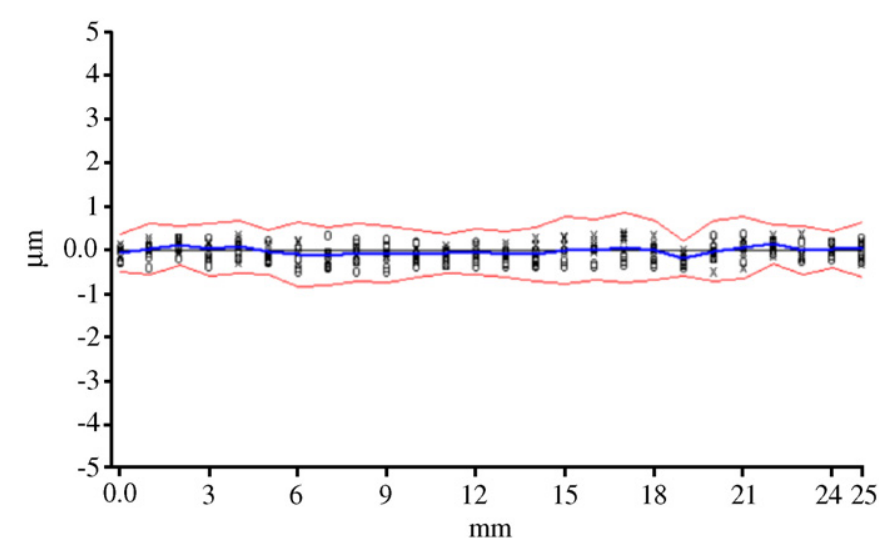

Fig. 19. Long stroke (AC mode) positioning accuracy of one axis. 
frequency range, yielding to poor reflective light intensity and poor $S$-curve.

In the experiments of autofocusing motion, the objective lens will be moved by the VCM along with the surface variation in order to lock the zero voltage of the 4-Q detector output. Fig. 22 shows the result that a $1.5 \mathrm{~mm}$ linear range of the servo-controlled displacement of the
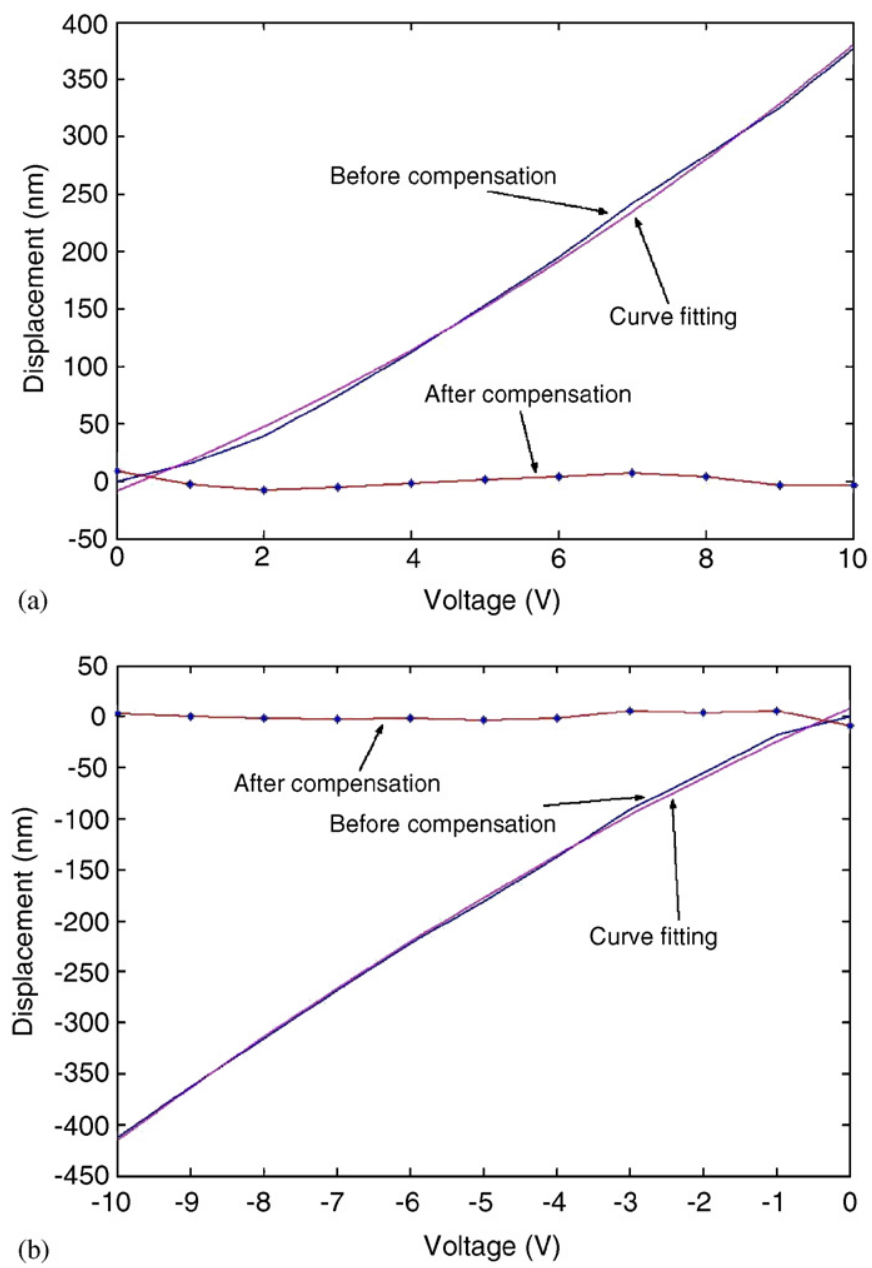

Fig. 20. Short stroke (DC mode) positioning accuracy: (a) forward; (b) backward. voice coil motor with respect to the FES was found [22]. Having calibrated, the probe can reach to around $0.1 \mu \mathrm{m}$ accuracy for autofocusing (Servo-FES) function and $30 \mathrm{~nm}$ accuracy for focusing (FES) function.

\subsection{System integration}

The whole software system of this prototype microCMM is developed in the commercially available LabVIEW 6.0 development system in association with the interface cards of National Instrument Co. Current work has not completed the system integration and tuning processes. Very laborious and patient task must be paid to the polish of the Teflon pad in order to ensure smooth and straight motion of each axis. Moreover, the volumetric errors of the CMM have to be calibrated and compensated in order to achieve higher accuracy. Accuracy is also sensitive to ambient conditions. An environmental-controlled chamber is under development to enclose this machine.

\section{Concluding remarks}

This article states the current progress on the development of a micro-CMM. Design considerations and preliminary results are described. Particular consideration is given to the structural accuracy, with an innovative archbridge and a co-planar stage proposed. With the particular

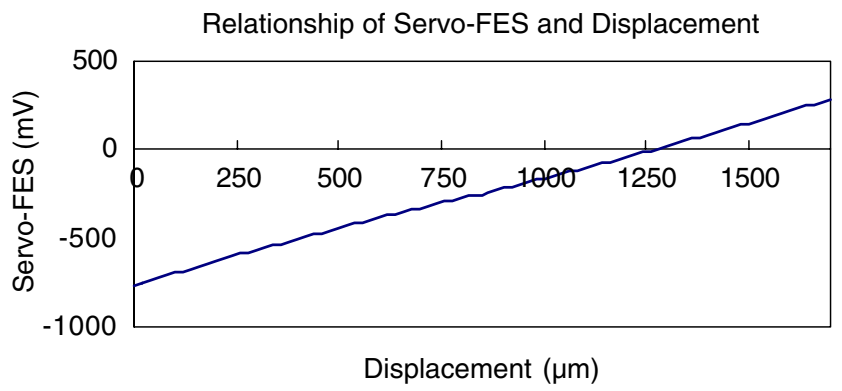

Fig. 22. Relationship between the motion of the objective lens and the FES.

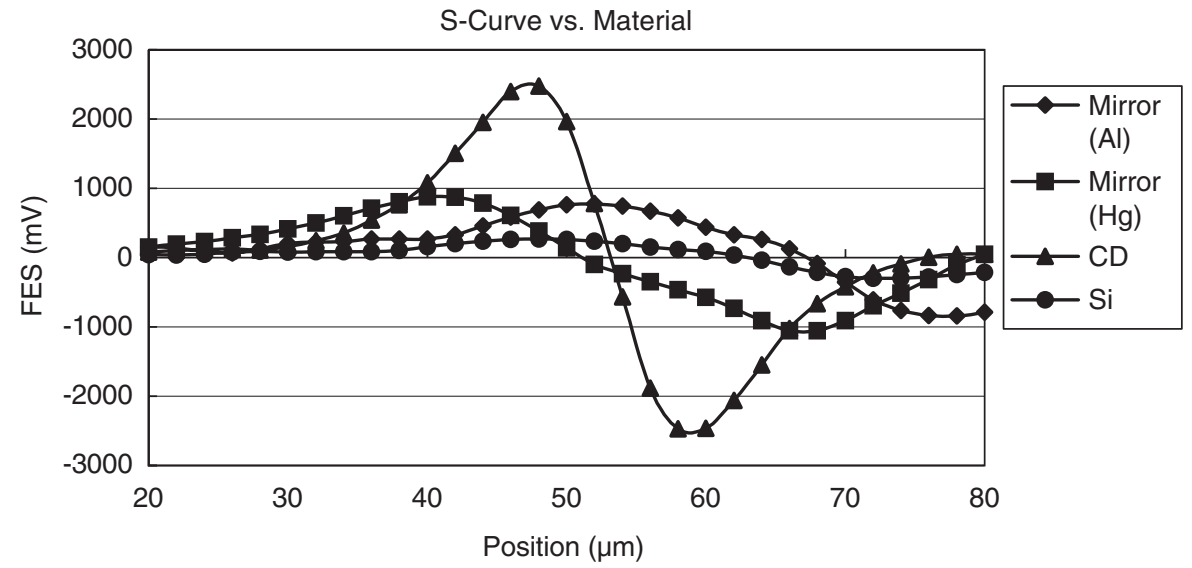

Fig. 21. S-curves of the autofocusing probe with respect to various materials. 
consideration in the structural accuracy, the innovative arch-bridge and the co-planar stage are proposed. The innovative arch-bridge performs better stiffness than the conventional rectangular bridge. Equipped with the ultrasonic actuator and the LDGI feed back position sensor the motion control of the stage in three directions can be achieved in both long stroke and fine motion modes. The autofocusing probe is integrated into the system to form a prototype non-contact micro-CMM with the accuracy within $30 \mathrm{~nm}$ for FES short-range detection (below $30 \mu \mathrm{m}$ ) and $0.1 \mu \mathrm{m}$ for long-range detection (up to $1 \mathrm{~mm}$ ). Experimental tests have shown the feasibility of the current system. So far, the required accuracy of $30 \mathrm{~nm}$ and resolution of $1 \mathrm{~nm}$ have been achieved on each axis motion under a normal temperature-controlled environment. Future tasks will implement the performance tests and volumetric error compensation to further enhance the system accuracy. In addition, more rigorously controlled working temperature and anti-vibration pad, named as "instrument mini environment chamber", will be developed in the future.

\section{Acknowledgments}

The present authors gratefully acknowledge the support provided to this cooperative project from the National Natural Science Council of China under Contract Nos. 50275048 and 50420120134, and the National Science Council of Taiwan under Contract No. 922212E002038.

\section{References}

[1] McKeown P. Nanotechnology-special article. Proceedings of the nano-metrology in precision engineering, Hong Kong; 1998. p. 5-55.

[2] Akamine S, Albrecht TR, Zdeblick MJ, Quate CF. Microfabricated scanning tunneling microscope. IEEE Electron Device Lett 1989; 10(1):490-2.

[3] Binning G, Quate CF, Gerber CH. Atomic force microscopy. Phys Rev Lett 1986;56(9):930-3.

[4] Deng KL, Wang JP. Nanometer-resolution distance measurement with non-interferometer method. Appl Opt 1994;33(1):113-6.

[5] Zhang JH, Cai LL. An autofocusing measurement system with piezoelectric translator. IEEE/ASME Trans Mechatronics 1997;2(3): $213-6$.
[6] Takamasu K, Furutani R, Ozuno S. Basic concept of feature-based metrology. Measurement 1999;26:151-6.

[7] Peggs GN, Lewis A, Leach RK. Measuring in three dimensions at the mesoscopic level. Proceedings of the ASPE winter topical meeting-machines and processes for micro-scale and meso-scale fabrication, metrology and assembly, FL, USA; 2003. p. $53-7$.

[8] Haitjema H, Pril WO, Schellekens P. Development of a silicon-based nanoprobe system for 3-D measurements. Ann CIRP 2001;50(1): 65-368.

[9] Schwenke H, Härtig F, Wendit K, Wäldele F. Future challenges in co-ordinate metrology: addressing metrological problems for very small and very large parts. Proceedings of IDW conference, Knoxville; 2001. p. 1-12.

[10] Jäger G, Grǔnwald R, Manske E, Hausote T, Fußl R. A nanopositioning and nanomeasuring machine, operation measured results. Nanotechnol Precis Eng 2004;2(2):81-4.

[11] Hocken RJ, Trumper DL, Wang C. Dynamics and control of the UNCC/MIT sub-atomic measuring machine. Ann CIRP 2001; 50(1):373-6.

[12] Fan KC, Chu CL, Chang SH, Chung TT. Development of a microCMM for nanometrology. Proceedings of KSPE spring conference, Korea; 2001. p. 1-8.

[13] Fan KC, Wang WL, Chen F. Innovative design of a new CMM bridge. Chin J Mech Eng 2004;17(2):170-3.

[14] Pohl DW. Dynamic piezoelectric translation devices. Rev Sci Instrum 1987;58(1):54-7.

[15] Chang SH, Du BC. A precision piezo-driven micropositioner mechanism with large travel range. Rev Sci Instrum 1998;69(4): 1785-91.

[16] Jourlin Y, Jay J, Parriaux O. Compact diffractive interferometric displacement sensor in reflection. Precis Eng 2002;26:1-6.

[17] Heydemann PLM. Determination and correction of quadrature correction of fringe measurement errors in interferometers. Appl Opt 1981;3382.

[18] Chen BY, Li DC. Progress in studies on long-range and ultrahighaccuracy manometer measurements. Proceedings of the second ISIST, Jinan China; 2002. p. 84-9.

[19] Wen P, Hsu DH. Direct subdivision of moire fringe with CCD. Proc SPIE 1990;1230:165-6.

[20] Jäger G, Manske E, Hausote T, Fußl R, Grǔnwald R, Buchner HJ. Micro and nano devices based on miniature plan mirror interferometers. Proceedings of the eighth international conference on mechatronics technology, Hanoi, Vietnam; 2004. p. 7-11.

[21] Fan KC, Lin CY, Shyu LH. Development of a low-cost focusing probe for profile measurement. Meas Sci Technol 2000;11(1):1-7.

[22] Fan KC, Chu CL, Mou JI. Development of a low-cost autofocusing probe for profile measurement. Meas Sci Technol 2001;12: 2137-46. 